\title{
RESISTENCIA A ANTIHELMÍNTICOS Y PREVALENCIA DE FASCIOLOSIS BOVINA EN LA GANADERÍA LECHERA DE JAUJA, PERÚ
}

\author{
Resistance to Anthelmintics and Prevalence of Bovine F asciolosis in \\ Dairy Farms in Jauja, Peru
}

Amanda Chávez V. ${ }^{1,4}$, Lilian Sánchez R. ${ }^{1}$, Carlos Arana D. ${ }^{3}$, Francisco Suárez A. ${ }^{2}$

\section{RESUMEN}

\begin{abstract}
El objetivo del estudio fue determinar la prevalencia de Fasciola hepatica mediante exámenes coprológicos en el ganado bovino del distrito de Huertas, provincia de JaujaJunín, y evaluar la eficacia de dos fasciolicidas ampliamente utilizados en la zona: triclabendazol (TBCZ) y albendazol (ABZ). Se trabajó con 387 vacas de 30 establos durante la época de lluvias. Se colectaron muestras de heces directamente del recto y se analizaron mediante los métodos de sedimentación espontánea y de Mc Master modificado. La prevalencia de $F$. hepatica fue de $38.2 \%$ con cargas promedio de 16 hpg (1-197 hpg). Asimismo, 26 establos (86.7\%) resultaron positivos a F. hepatica. En la evaluación de resistencia a las drogas se empleó la prueba de reducción de recuento de huevos (FECRT). Se seleccionaron 75 animales que superaron recuentos de 13 hpg y se distribuyeron aleatoriamente en dos grupos: a) TCBZ, $12 \mathrm{mg} / \mathrm{kg}$ peso vivo ( $\mathrm{n=39}$ ) y b) ABZ, 10 $\mathrm{mg} / \mathrm{kg}$ peso vivo ( $\mathrm{n}=33$ ). Las drogas fueron administradas vía oral. La eficacia del TBCZ a los 7, 14, 21 y 28 días post tratamiento fue de 53.4, 53.3, 36.4 y 34.9\%, respectivamente, mientras que para el ABZ fue nula en todos los casos. Se concluye que la ganadería lechera de Jauja, Junín, muestra una alta prevalencia de F. hepatica, además, de presentar resistencia del parásito al albendazol y triclabendazol.
\end{abstract}

Palabras clave: fasciolosis, bovino, tremátodo, resistencia antihelmíntica

\section{AbSTRACT}

The objective of this study was to determine the prevalence of Fasciola hepatica through coprological analysis in dairy cattle in the district of Huertas, province of Junin, Peru and to evaluate the efficacy of two anthelmintics frequently used in the region:

\footnotetext{
${ }^{1}$ Laboratorio de Microbiología y Parasitología Veterinaria, ${ }^{2}$ Laboratorio de Medicina Veterinaria Preventiva, Facultad de Medicina Veterinaria, Universidad Nacional Mayor de San Marcos, Lima

${ }^{3}$ Centro de Investigación IVITA - El Mantaro, Huancayo

${ }^{4}$ E-mail: achavezvg@gmail.com
} 
triclabendazol (TBCZ) and albendazol (ABZ). Faecal samples were collected from 387 cows in 30 farms. Samples were analysed by the spontaneous sedimentation test and the modified Mc Master test. The prevalence of F. hepatica was 38.2\%, with a mean of 16 eggs per gram of faeces (epg) and a range of 1 to 197 epg. Furthermore, 26 farms (86.7\%) were positive to $F$. hepatica. The faecal egg count reduction test (FECRT) was used for evaluating the parasite resistance to the two anthelmintics. For this, 75 animals with more than 12 epg were selected and randomly allocated to two groups: a) TCBZ, $12 \mathrm{mg} / \mathrm{kg}$ body weight ( $\mathrm{n=39}$ ) and b) ABZ, $10 \mathrm{mg} / \mathrm{kg}$ body weight $(\mathrm{n}=33)$. Both drugs were orally administered. The efficacy of TBCZ on days 7, 14, 21, and 28 was 53.4, 53.3, 36.4, and $34.9 \%$ respectively, whereas ABZ was zero in all cases. It is concluded that dairy cattle in Huertas, Jauja has a high prevalence of $F$. hepatica and the parasite shows resistance to albendazol and triclabendazol.

Key words: fascioliosis, bovine, trematode, anthelmintic resistance

\section{INTRODUCCIÓN}

La distomatosis, ocasionada por Fasciola hepatica, constituye la segunda enfermedad parasitaria en importancia en la ganadería del Perú (Leguía, 1988), llegándose a estimar pérdidas económicas de $50 \mathrm{mi}$ llones de dólares al año debido a pérdidas por el decomiso de órganos en el camal, la baja ganancia de peso y menor fertilidad, así como por los costos asociados al tratamiento; sin embargo, es difícil estimar tanto el impacto económico de la fasciolasis humana (Espinoza et al., 2010) como los problemas de salud que le ocasiona al hombre (MasComa et al., 1999).

Dentro de las drogas más efectivas para el control de fasciolosis se halla al triclabendazole (TCBZ), el cual ha sido ampliamente aceptado a partir de la década del 80 , constituyéndose el de mayor uso a nivel mundial para el control de la distomatosis. Diversos estudios en ovinos han demostrado una eficacia de $100 \%$ en infecciones con $F$. hepatica de 6 semanas a dosis de $10 \mathrm{mg} / \mathrm{kg}$ (Boray et al., 1983), de 99\% en infecciones de 1 a 12 semanas (Smeal y Hall, 1983), y de 99-100\% en infecciones de 4 a 14 semanas (Boray, 1997). Así mismo, el tratamiento de elección actual para la fasciolosis humana es el triclabendazol; aunque en el Perú se utiliza con frecuencia el producto comercial de uso veterinario, dado que la formulación para uso humano no se encuentra ampliamente disponible (Maco et al., 2002). Por otro lado, estudios sobre la eficacia del albendazol (ABZ) en formas adultas de $F$. hepatica en terneros con dosis de $10 \mathrm{mg} / \mathrm{kg}$, mostraron una eficacia adulticida de $85.9 \%$, así como reducción en el recuento de huevos en heces del 95.6\% (Malone et al., 1982).

El espectro de eficiencia de las drogas fasciolicidas es muy variado. Algunas no son efectivas para estados inmaduros de parásitos menores de ocho semanas y son justamente aquellos estados que constituyen el mayor perjuicio para el hospedero (Leguía, 1988). Al utilizarse fasciolicidas para parásitos adultos se tiene la desventaja de tener que repetir el tratamiento porque las fases juveniles que están migrando por el parénquima hepático no son afectadas y estas darán lugar a parásitos adultos en los conductos biliares en el corto plazo.

Por otra parte, se ha creado resistencia a muchos fasciolicidas debido a la subdosificación, la falta de alternancia de fasciolicidas, a desparasitaciones frecuentes, etc. Se han descrito fenómenos de resistencia frente a clorsuron, albendazol y triclabendazol (Vara-Del Rio et al., 2006), albendazol y rafoxanide (Elitok et al., 2006) 
y triclabendazol (Moll et al., 2000; Mooney et al., 2009), los cuales son los fasciolicidas de mayor uso a nivel mundial (Mas-Coma et al., 1999).

El objetivo del estudio fue determinar la prevalencia de $F$. hepatica en el ganado bovino de establos lecheros en un distrito del departamento de Junín, mediante exámenes coprológicos; así como evaluar la efectividad de los dos antihelmínticos más utilizados en la zona: albendazol y triclabendazol.

\section{Materiales y MéTOdos}

\section{Fundos Ganaderos y Animales}

El estudio se realizó en 30 establos ganaderos del distrito de Huertas, ubicado en la provincia de Jauja, departamento de Junín, Perú. La zona presenta un clima templado seco, con una temperatura media anual de $11.1^{\circ} \mathrm{C}$ y una altitud de cercana a los 3380 msnm. La época de lluvias corresponde al periodo entre los meses de noviembre y abril, y el estudio se realizó entre enero a marzo de 2009.

Las vacas del estudio fueron mayormente cruces entre Brown Swiss o Holstein con ganado criollo, con edades entre 2 a 8 años, peso vivo de 280 a 500 kg y condición corporal de 2 a 3.5 en una escala de 0 a 5 . Los animales no habían recibido dosificación antihelmíntica al menos dos meses antes del inicio del estudio. Los animales fueron mantenidos en pasturas naturales y cultivadas, $\mathrm{y}$ el agua de bebida la obtenían de fuentes naturales como riachuelos y acequias.

El tamaño muestral $(\mathrm{n}=387)$ se determinó mediante la fórmula de proporciones infinitas (Daniel, 1996), pero los animales fueron seleccionados por conveniencia de muestreo, y no en forma proporcional al número de animales que existía en los establos, dada la gran variabilidad que existía (entre 5 a 40 animales por establo).
Dos semanas previas a la fase experimental se colectaron muestras de heces del recto (40-50 g) de 387 vacas de los 30 establos de la zona. Las muestras se guardaron en recipientes térmicos con refrigerantes y fueron trasladadas para su análisis al Laboratorio de Parasitología de la Facultad de Medicina Veterinaria, Universidad Nacional Mayor de San Marcos, Lima.

En la evaluación coproparasitológica se utilizó el método de sedimentación espontánea (Tello, 1988) para determinar la presencia de huevos de F. hepatica. En las muestras positivas se hizo el contaje de huevos por gramo de heces (hpg) mediante la técnica de Mc Master modificado (Conceição et al., 2002).

\section{Grupos Experimentales}

Se utilizaron los animales positivos a $F$. hepatica, cuyos recuentos individuales superaban los 13 hpg para evaluar la eficacia de los fasciolocidas. Se escogieron dos animales por establo como mínimo, los que fueron distribuidos aleatoriamente en los dos grupos experimentales.

Las drogas fasciolicidas y dosis empleadas por tratamiento fueron: a) Triclabendazole (TBCZ) en dosis de $12 \mathrm{mg} / \mathrm{kg}$ peso vivo; b) Albendazole (ABZ) en dosis de $10 \mathrm{mg} / \mathrm{kg}$ peso vivo. En ambos casos se emplearon las dosis recomendadas por los laboratorios fabricantes. La determinación del peso vivo de los animales se hizo mediante una cinta bovinométrica comercial. Las muestras de heces se recolectaron los días 0 (fecha de inicio y administración de las drogas), 7, 14, 21 y 28 post tratamiento.

El experimento se inició con 45 animales por grupo experimental, donde las cargas parasitarias fueron similares; sin embargo, los propietarios de los animales vendieron o redosificaron algunos animales, lo cuales tuvieron que ser descartados del estudio. Esto significó que el análisis de los resultados se haga 
con 39 animales en el grupo TCBZ y 33 en el grupo ABZ, causando un desbalance en las cargas parasitarias iniciales (65.9 para TCBZ y 28.0 para ABZ).

\section{Análisis Estadístico}

La prevalencia de fasciolosis se expresó en porcentajes de positividad a F. hepática con intervalos de confianza del 95\%. La eficacia antihelmíntica se realizó mediante el cálculo de la reducción en el recuento de huevos por gramo de heces o prueba de reducción de recuento de huevos (FECRT), aplicando la fórmula: Diferencia entre el promedio de hpg del día 0 con el promedio de hpg de los días posteriores al tratamiento $(7,14,21,28)$, dividido por el promedio de hpg del día 0 , y expresado como porcentaje.

Considerando que el número de huevos no sigue una distribución normal, los datos fueron transformados en forma logarítmica, para determinar el promedio y rango del número de hpg por grupo. Las diferencias en hpg por animal entre el día 0 con los días 7 , 14, 21 y 28 del tratamiento se analizaron mediante una prueba pareada de análisis de varianza, empleando el software estadístico STATA. El nivel de significación estadística fue de 0.05 .

Eficacias mayores de 90\% indicaban que la droga utilizada era efectiva; eficacias entre 80 y $89 \%$ indicaban que son moderadamente efectivas, y eficacias menores de $80 \%$ indicaban que son insuficientemente efectivas y la resistencia debería ser considerada como un problema (Kassai, 1998).

\section{Resultados}

Los análisis coprológicos indicaron que el 86.7\% (26/30) de establos presentaron al menos un animal con huevos de F. hepatica. La prevalencia de este parásito fue de $38.2 \%$ (148/387), con un promedio de 16 hpg y un rango de cargas de 1 a 197 hpg en los animales que se hallaban eliminando huevos de este trematodo.
En el Cuadro 1 se observan los porcentajes de reducción de huevos en heces (FECRT) durante los 28 días posteriores al tratamiento. En el caso de TCBZ se obtienen los valores de reducción más altos los días 7 y 14 con 53\%, mientras que en el caso de ABZ los valores fueron negativos ya que el número de huevos fue en aumento.

\section{Discusión}

La técnica de sedimentación posee una especificidad del $100 \%$ pero una sensibilidad de $70 \%$ en un examen, pero se incrementa cuando se hacen exámenes seriados a un mismo animal (Quiroz, 2000). Esta relativa baja sensibilidad podría dejar un margen de casos positivos no detectados y, por lo tanto, se podría estar frente a un porcentaje de animales falsos negativos que incrementarían el $86.7 \%$ de establos afectados y el $38.2 \%$ de prevalencia de $F$. hepatica encontrado en el presente estudio, lo cual denotaría aún más el carácter endémico de este parásito en la localidad.

Soulsby (1993) sostiene que cargas de 100 a 200 hpg en bovinos son consideradas graves, mientras que Kassai (2002) señala que cargas de 10 a 25 hpg reflejan infecciones de grado intermedio. Los bovinos del distrito de Huertas reciben periódicamente tratamientos para controlar la distomatosis, por lo que el nivel de prevalencia hallado y el promedio de carga parasitaria demuestran que esta parasitosis no está siendo debidamente controlada a pesar de los tratamientos impartidos.

Huertas es un distrito dedicado a la crianza de ganado lechero, pero no se han realizado estudios previos sobre prevalencia de distomatosis. No obstante, en un trabajo realizado con cerca de 30000 bovinos beneficiados en 2005 en camales municipales del departamento de Junín se encontró el 41.1\% de animales afectados con distomatosis (Espinoza et al., 2010). Otros reportes en el país señalan la presencia de la enfermedad 
Cuadro 1. Eficacia del tratamiento del triclabendazole (TCBZ; $\mathrm{n}=39$ ) y albendazole (ABZ; $\mathrm{n}=33$ ) en vacas infectadas naturalmente con Fasciola hepatica en la Sierra de Junín (2009)

\begin{tabular}{|c|c|c|c|c|c|c|}
\hline \multirow{2}{*}{$\begin{array}{l}\text { Días post } \\
\text { tratamiento }\end{array}$} & \multicolumn{3}{|c|}{ TCBZ } & \multicolumn{3}{|c|}{$\mathrm{ABZ}$} \\
\hline & $\begin{array}{c}\text { Promedio } \\
\text { (hpg) }\end{array}$ & $\begin{array}{l}\text { Rango } \\
\text { (hpg) }\end{array}$ & $\begin{array}{c}\text { Eficacia } \\
(\%)\end{array}$ & $\begin{array}{l}\text { Promedio } \\
\text { (hpg) }\end{array}$ & $\begin{array}{l}\text { Rango } \\
\text { (hpg) }\end{array}$ & $\begin{array}{c}\text { Eficacia } \\
(\%)\end{array}$ \\
\hline 0 & 65.9 & $13-320$ & & 28.0 & 13-93 & \\
\hline 7 & 30.7 & $4-126$ & 53.4 & 34.0 & $9-106$ & 0 \\
\hline 14 & 30.8 & $11-115$ & 53.3 & 35.0 & $15-109$ & 0 \\
\hline 21 & 41.9 & $13-198$ & 36.4 & 45.0 & $11-131$ & 0 \\
\hline 28 & 42.9 & $0-181$ & 34.9 & 59.0 & $17-163$ & 0 \\
\hline
\end{tabular}

con prevalencias moderadas a elevadas; así, $10 \%$ en Pasco, 22\% en Huánuco, 39\% en Junín, 43\% en Huancavelica (Manrique y Cuadros, 2002); 10\% en Oxapampa (Páucar et al., 2010), y 36\% en Ayacucho (Ticona et al., 2010).

Los resultados del estudio son preocupantes, toda vez que los ganaderos de la zona vienen utilizando frecuentemente drogas comerciales, sin aplicar un tratamiento estratégico y guiándose muchas veces por la sintomatología manifestada por el animal (diarrea, enflaquecimiento, etc.) y los costos de las drogas comerciales. Además, reportes de hace una década realizados en el distrito de Huertas por Marcos et al. (2002), señalaron una prevalencia de $27.2 \%$ de niños afectados con este trematodo, y todo indicaría que estos valores no se hayan modificado en la actualidad, dado que no existe una política para su control. El hecho de haberse hallado problemas de resistencia contra TCBZ constituiría un grave problema en Salud Pública, toda vez que dicha droga es utilizada en el tratamiento de las personas, tanto en su presentación de uso humano como de uso ve- terinario (Mas-Coma et al., 1999; Talaie et al., 2004).

En las zonas ganaderas de la provincia de Jauja se utiliza con frecuencia tanto el ABZ como el TCBZ para el control de $F$. hepatica. El primero constituye un adulticida, dado que su mayor efectividad se presenta en distomas de 10 semanas (Malone et al., 1882; Fairweather y Boray, 1999), mientras el TCBZ es reconocido por su alta efectividad en formas maduras e inmaduras (Boray et al., 1983).

El tratamiento con TCBZ mostró ser más eficaz los días 7 y 14 post tratamiento, no obstante que los mejores resultados se esperarían hallar entre los 14 y 21 días post tratamiento (Elitok et al., 2006). Por otro lado, los resultados de eficacia de TCBZ fueron inferiores al valor mínimo considerado como moderadamente efectivos (Kassai, 1998), mientras que el tratamiento con ABZ mostró una nula eficacia en la reducción de recuentos de huevos. Flanagan et al. (2011), también pudieron demostrar resistencia de $F$. hepatica a ambos fasciolicidas mediante FECRT. 
La presencia de resistencia de $F$. hepatica al tratamiento con antihelmínticos en bovinos ha sido reportado en varios países. Así, se ha demostrado cuadros de resistencia para ambas drogas en España (Álvarez-Sánchez et al., 2006)., para ABZ en Turquía y España (Elitok et al, 2006; VaraDel Río et al., 2006), y para TCBZ en Holanda (Moll et al., 2000), Irlanda (Mooney et al., 2009) y, recientemente, en la Patagonia de Argentina (Olaechea et al., 2011). En el Perú existen reportes técnicos sobre este problema en Cajamarca (Moncada, 2006).

La baja eficacia de los antihelmínticos ocurre fundamentalmente como respuesta a tratamientos frecuentes con los mismos productos (o de familias idénticas) y por cálculos incorrectos de dosis o uso de dosis incompletas con fines de ahorro (subdosificaciones). La elaboración de nuevos antihelmínticos que sean eficaces resulta muy costoso y toma bastante tiempo, por lo que se debe evitar el desarrollo de resistencia a los antihelmínticos disponibles.

Para evitar la aparición de resistencias debe combinarse un diagnóstico temprano con una rotación adecuada de antihelmínticos, dosificaciones con dosis correctas, y la aplicación de tratamientos estratégicos sobre la base de estudios epidemiológicos de distomatosis. Estas medidas, muchas veces subestimadas, podrían reducir los niveles de infección, así como la frecuencia de los tratamientos. Asimismo, la combinación de fármacos con diferentes modos de acción puede ser eficaz contra cepas resistentes de $F$. hepatica, aunque este enfoque tiene el riesgo de crear resistencia a múltiples fármacos (Gaasenbeek et al., 2001).

\section{ConClusiones}

- La prevalencia de Fasciola hepatica determinada mediante exámenes coproparasitológicos en los establos lecheros del distrito de Huertas fue de $38.2 \%$, con una carga de 16 hpg (1-197 hpg).
- Se encontró resistencia de Fasciola hepatica a las drogas albendazol y triclabendazol.

\section{Agradecimiento}

El presente estudio fue financiado por el Consejo Superior de Investigaciones de la Universidad Nacional Mayor de San Marcos (Código 090801141).

\section{Literatura Citada}

1. Álvarez-Sánchez MA, Mainar-Jaime RC, Pérez-García J. Rojo-Vázquez FA. 2006 Resistance of Fasciola hepatica to triclabendazole and albendazole in sheep in Spain. Vet Rec 159: 424-425.

2. Boray JC. 1997. Chemotherapy of infections with Fasciolidae. In: Boray JC (ed). Immunology, pathobiology and control of Fasciolosis. New Jersey, USA: MSD AGVET. p 83-97.

3. Boray JC, Crowfoot PD, Strong MB, Allison JR, Schellenbaum M, Von Orelli M, Craigand Huey R. 1983. Treatment of immature and mature Fasciola hepatica infections in sheep with triclabendazole. Vet Rec 113: 315-317.

4. Conceição M, Durão R. CI, Correia da Costa J. 2002. Evaluation of a simple sedimentation method (modified McMaster) for diagnosis of bovine fascioliosis. Vet Parasitol 105: 337-343.

5. Elitok B, Elitok OM, Kabu M. 2006. Field trial on comparative efficacy of four fascio-licides against natural liver fluke infection in cattle. Vet Parasitol 135: 279-285.

6. Espinoza JR, Terashima A, HerreraVelit P, Marcos LA. 2010. Fasciolosis humana y animal en el Perú: impacto en la economía de las zonas endémicas. Rev Peru Med Exp SaludPública 27(4): 604-612.

7. Fairweather I, Boray JC. 1999. Fasciolicides: efficacy, actions, resistance and its management. Vet J 158: 81-112. 
8. Flanagan A, Edgar HW, Gordon A, Hanna RE, Brennan GP, Fairweather I. 2011. Comparison of two assays, a fecal egg count reduction test (FECRT) and a coproantigen reduction test (CRT), for the diagnosis of resistance to triclabendazole in Fasciola hepatica in sheep. Vet Parasitol 176: 170-176.

9. Gaasenbeek CP, Moll L, Cornelissen JB, Vellema P, Borgsteede FH. 2001. An experimental study on triclabendazole resistance of Fasciola hepatica in sheep. Vet Parasitol 95: 37-43.

10. Kassai T. 1998. Helmintología veterinaria. Zaragoza: Acribia. 258 p.

11. Leguía G. 1988. Distomatosis hepática en el Perú: epidemiología y control. Lima: Ciba Geigy-Hoesch. 42 p.

12. Maco V, Marcos LA, Terashima MA, Samalvides F, Espinoza JR, Gotuzzo E. 2002. Efficacy and tolerability of two therapeutic regimes of Triclabendazole (FASINEX) for the treatment of human fascioliasis in Perú. L Annual Meeting of the American Society of Tropical Medicine and Higiene 67(2): 232-233.

13. Malone JB, Smith PH, Loyacano AF, Hembry FG, Brock LT. 1982. Efficacy of albendazole for treatment of naturally acquired Fasciola hepatica in calves. Am J Vet Res 43: 879-881.

14. Manrique MJ, Cuadros CS. 2002. Fasciolosis: Buscando estrategias de control. Arequipa, Perú: Akuarella. 126 p.

15. Marcos RL, Maco FV, Terashima IA, Samalvides CF, Gotuzzo HE. 2002. Prevalencia de parasitosis intestinal en niños del valle del Mantaro, Jauja, Perú. Rev Med Hered 13(3): 85-89.

16. Mas-Coma S, Bargues MD, Esteban JG. 1999. Human fasciolosis. Dalton JP (ed). Human Fasciolosis. Wallingford, United Kingdom: CABI Pub. 544 p.

17. Moll L, Gaasenbeek CP, Vellema $P$, Borgsteede FH. 2000. Resistance of Fasciola hepatica against triclabendazole in cattle and sheep in the Nethrelands. Vet Parasitol 91: 153-158.
18. Moncada JR 2006. Efectividad y resistencia antihelmíntica de Fasciola hepatica a triclabendazol en el fundo "El Cortijo”, distrito Baños del IncaCajamarca, Perú. [Internet]. Disponible en: http://www.engormix.com/MAganaderia-carne/sanidad/articulos/efectividad-resistencia-antihelminticafasciola-t1421/p0.htm

19. Mooney L, Good B, Hamraham JP, Mulcahy G, de Waal T. 2009. The comparative efficacy of four anthelmintics against a natural acquired Fasciola hepatica infection in hill sheep flock in the west of Ireland. Vet Parasitol 164: 201-205.

20. Olaechea F, Lovera V, Larroza M, Raffo F, Cabrera R. 2011. Resistance of Fasciola hepatica against triclabendazole in cattle in Patagonia (Argentina). Vet Parasitol 178: 364-366.

21. Páucar SS, Chávez AV, Casas EA, Suárez FA. 2010. Prevalencia de fascioliasis y paramfistomiasis en el ganado lechero de Oxapampa, Pasco. Rev Inv Vet, Perú 21: 87-92.

22. Quiroz HR. 2000. Parasitología y enfermedades parasitarias de animales domésticos. México DF: Uteha. 460 p.

23. Smeal MG, Hall CA. 1983. The activity of triclabendazole against immature and adult Fasciola hepatica infections in sheep. Austr Vet J 60: 329-331.

24. Soulsby EJL. 1993. Parasitología y enfermedades parasitarias en los animales domésticos. $7^{\mathrm{a}}$ ed. México DF: Interamericana. $820 \mathrm{p}$.

25. Talaie H, Emami H, Yadegarinia D, Nava-Ocampo A, Massoud $J$, Azmoudeh M, Mas-Coma S. 2004. Randomized trial of a single, double, and triple dose of $10 \mathrm{mg} / \mathrm{kg}$ of a human formulation of triclabendazole in patients. Clin Exp Pharmacol Physiol 31: 777-782.

26. Tello R. 1988. Empleo de una nueva técnica parasitológica rápida sedimentación espontánea en el diagnóstico de protozoarios y helmintos. V Jornadas Científicas. Lima: UPCH. 
27. Ticona DS, Chávez AV, Casas GV, Chavera AC, Li OE. 2010. Prevalencia de Fasciola hepatica en bovinos y ovinos de Vilcashuamán, Ayacucho. Rev Inv Vet, Perú 21: 168-174.

28. Vara-Del Río MP, Martínez-Valladares M, Martínez-Delgado A, RojoVázquez FA. 2006. Prevalencia de la fasciolosis ovina y estudios de resistencias a fasciolicidas en la provincia de León. En: Veinte años de Buiatría: Actas del XIV Congreso Internacional de la Federación Mediterránea de Sanidad y Producción de Rumiantes. Lugo, España. p 523-529. 